\title{
Masculinidad y paternidad: los riesgos en la salud a partir de los aprendizajes de género
}

\author{
Masculinity and fatherhood: \\ Health risks from gender learning
}

\author{
Alejandra Salguero Velázquez, Diana Córdoba Basulto \\ y Salvador Sapién López ${ }^{1}$
}

\begin{abstract}
RESUMEN
El objetivo del presente trabajo fue analizar los riesgos en la salud de los varones en México a partir de los aprendizajes de género sobre la masculinidad y la paternidad. Se incorpora una revisión teórica de esos aprendizajes y estereotipos, que no consideran el cuidado de la salud ni la revisión médica o psicológica en las rutinas masculinas porque pondría en evidencia su vulnerabilidad, lo que coloca a los hombres en condiciones de riesgo para la salud y hace que experimenten afecciones que se manifiestan en estados de ansiedad, angustia, depresión y en ocasiones suicidio.
\end{abstract}

Palabras clave: Masculinidad; Paternidad; Salud; Estereotipos de género; Hombres.

\begin{abstract}
The aim of the present study was to analyze health risks in Mexican men linked to gender learning about masculinity and paternity. The study incorporates a theoretical revision on such learning and gender stereotypes. The analysis indicates that such learning does not involve men's health care nor the medical or psychological revision of their routines, probably because it would expose their vulnerability, and would place them in risky health conditions, including symptoms of such ailments as anxiety, anguish, depression and in some cases, suicide.
\end{abstract}

Key words: Masculinity; Fatherhood; Health; Gender stereotypes; Men.

\section{INTRODUCCIÓN}

$\mathrm{P}$ odría comenzar el presente ejercicio teórico a partir de algunas interrogantes como las siguientes: ¿Es necesario hablar sobre las condiciones de riesgo en la salud de los hombres en México? ¿Es posible relacionar la masculinidad y la paternidad con algunas condiciones de salud? ¿Cuáles son las preocupaciones y temores en su condición de hombres y padres? ¿Hay desventajas sociales a partir de los roles estereotipados de género? Éstas y otras interrogantes condujeron a elaborar el presente trabajo bajo la premisa de que los aprendizajes y estereotipos de género señalados plantean que los hombres son el sexo fuerte, que siempre deben asumir la responsabilidad y tener todo bajo control, que viven en condiciones favorables y ventajosas, y que, por tanto, ejercen una posición de poder en un mundo socialmente estructurado.

\footnotetext{
${ }^{1}$ Carrera de Psicología, Facultad de Estudios Profesionales Iztacala, Universidad Nacional Autónoma de México, Avenida de los Barrios No. 1, Los Reyes Iztacala, 54090 Tlalnepantla, Edo. de México, México, correos electrónicos: alevs@unam.mx, dicordoba@hotmail.com y josesapien@hotmail.com. Artículo recibido el 8 de mayo y aceptado el 3 de julio de 2017.
} 
Parecía necesario seguir indagando sobre los aprendizajes de género de los hombres en México: cómo aprendieron a ser hombres y qué tensiones representa asumir la responsabilidad de la familia, el trabajo o la proveeduría, aun cuando sus parejas aporten económicamente lo mismo o, en ocasiones, incluso más.

El sentido de responsabilidad se incorpora en la identidad de la mayoría de los hombres desde temprana edad, lo que los lleva a soportar, incluso a costa de su salud, condiciones y riesgos físicos y emocionales porque así lo indican los aprendizajes y estereotipos de género, que definen a los hombres por su fortaleza, dureza y aguante.

Dentro del concepto de salud habría que considerar a los hombres como una entidad separada, pues desde el punto de vista sociológico que relaciona el estado de funcionalidad con el rol socialmente asignado, la salud es una condición necesaria para el cumplimiento de las expectativas sociales. Parsons (1881) define la salud como "el estado de capacidad óptimo de un individuo para la realización efectiva de los roles y las tareas para los que ha sido socializado" (p. 57). El punto aquí sería el cuestionamiento respecto de los roles sociales y las tareas asignadas para el cumplimiento de los mismos. En el caso de los hombres implicados en su rol de padres, lo más valorado y exigido es la proveeduría, sin importar la forma a través de la cual la cubran ni las consecuencias para la salud que de ella se generen, como los tiempos mínimos para la convivencia familiar, los malestares y otras afecciones de la salud.

Desde el punto de vista psicológico sería pertinente investigar los estados emocionales, el manejo de los sentimientos, el estrés y la ansiedad asociados a las condiciones de vida, en que los aprendizajes y estereotipos del género masculino tienen fuertes implicaciones en las condiciones de salud, así como analizar como una posibilidad la vivencia de ser hombre y padre en condiciones saludables, tanto física como psicológicamente, lo cual sería posible si se lleva el concepto de salud más allá del modelo médico al incorporar no solo el estado físico sino también el psicológico, pues existe entre ambos una estrecha interrelación y afectación mutua (Figueroa y Nájera, 2015; Sánchez, 2000).
Psicológicamente, el concepto de salud se debe ver de manera integral, considerando a la persona como un todo, identificando de manera conjunta con ella los procesos a través de los cuales construye situaciones o estados de malestar consigo misma o con otras personas en la trayectoria de vida, así como el proceso terapéutico de acompañamiento a los varones para identificar y construir el estado de bien-estar a partir de las condiciones en las que se encuentren y los recursos socioculturales de que dispongan. Un compromiso desde la psicología de la salud es analizar, identificar, diagnosticar y abordar junto con los varones los procesos de desarrollo emocional, las dificultades y problemas que enfrentan y las maneras en que los resuelven.

La salud psicológica incorpora la parte emocional en el proceso de aprendizaje y construcción de ser hombre. Quienes han recibido atención psicológica se involucran en un proceso en el que elaboran y reelaboran momentos y experiencias de vida, nombrando aquello que han silenciado durante años, trabajando y resignificando experiencias dolorosas o conflictivas para encontrarles sentido y así elaborar nuevos significados que les permitan continuar, construir y reconstruir sus trayectorias como hombres, parejas o padres.

En algunos casos, los hombres, aun cuando parecen "estar bien", sufren en silencio y experimentan sentimientos de confusión, aislamiento, desesperanza, depresión o soledad que persisten de la infancia a la vida adulta, y que, cuando son padres, se llegan a agudizar por la responsabilidad que implica hacerse cargo de la vida de sus hijos.

Es a través de los aprendizajes de género como se incorporan estereotipos que los conducen a ocultar sus emociones y sentimientos, pues externarlos "no es de hombres". Figueroa (2001) habla así de "la soledad en la paternidad" para dar cuenta de las experiencias que muchos hombres no se atreven a manifestar porque se resisten a cuestionar ciertos modelos dominantes de masculinidad, que en el caso de la paternidad refieren a la pérdida de experiencias potencialmente gratificantes y lúdicas debido a la rigidez de los aprendizajes de género excluyentes y limitantes, con las consiguientes implicaciones en su salud física y emocional. 


\section{MASCULINIDAD: LOS RIESGOS A PARTIR DE LOS APRENDIZAJES Y ESTEREOTIPOS DE GÉNERO}

Hacer patentes las desventajas de los aprendizajes de género desde los cuales los hombres construyen sus identidades como tales conduce a indagar la manera a través de la cual los adquirieron. En muchos casos fue en las familias de origen en las que se señalaba que un hombre es quien "debe" mandar, tener el control de las situaciones, ser responsable y silenciar las emociones y sentimientos, pues eso corresponde a las mujeres, no a los hombres. Nolasco (1989) plantea que hay un principio masculino construido socialmente a partir del cual los hombres intentarán casi siempre administrar sus afectos. Este principio, base de la identidad masculina, segmenta y polariza lo femenino de lo masculino, lo sexual de lo afectivo, el trabajo del placer, convirtiendo así a los hombres en individuos divididos que viven inmersos en la ilusión de la perfección. Rivera y Ceciliano (2005) consideran que una parte de la representación social del ser hombre incorpora la idea de que debe evitar las emociones, sobre todo aquellas que son "típicamente femeninas", como demostrar cariño, afecto o miedo; ante todo, los hombres deben ser racionales y están para proteger, trabajar, proveer y ser fuertes y decididos. Fuller (2000) y Rodríguez (2001) señalan que los hombres construyen su identidad en un ámbito plagado de estereotipos, en una sociedad con predominio y privilegios masculinos, cuya conservación y consolidación se convierte en una parte y un cometido importantes de su existencia. El orden social de género acentuará esas dimensiones estereotipadas al maximizar las diferencias entre hombres y mujeres como grupos excluyentes (Connell, 2015).

Los estereotipos de género son una descripción simplificada de la realidad que reafirma la dimensión normativa para regular formas de conducta y actitudes; quienes no siguen dichas normas son socialmente sancionados y reprobados (Camussi y Leccardi, 2005). La evaluación o autoevaluación de las diferencias genéricas tiene implicaciones en las concepciones de realidad y del mundo de pertenencia. Cuando alguien se atreve a ser diferente o rompe con las expectativas este- reotipadas, recibe consecuencias por su conducta "desviada". Esas expectativas estereotipadas no son simplemente creencias y percepciones acerca de diferentes cualidades del hombre y la mujer, sino que adquieren un valor normativo y prescriptivo que identifica las características y conductas consideradas como "deseables" en las mujeres y hombres, así como en las mujeres-madres o en los hombres-padres. Tal dimensión involucra, pues, la formulación de juicios y apreciaciones que constituyen delimitaciones sobre lo que es correcto o deseable. Los estereotipos son prejuicios y generalizaciones con dimensiones normativas sobre lo que supone deben ser los hombres y las mujeres dentro de un orden "natural", exageraciones, e incluso ficciones, que ayudan a justificar el orden social existente; simplifican la realidad para el propósito de adaptación, y validan las diferencias de poder entre hombres y mujeres muchas veces mediante explicaciones naturalistas que afectan las posibilidades de vida.

Un caso particular son las condiciones y riesgos a la salud de los varones, particularmente por la falta del cuidado de sí mismos, lo que puede llegar a considerarse como una forma de violencia de acuerdo a la Organización Panamericana de la Salud (2002). En su Informe Mundial sobre la Violencia y la Salud, la Organización Mundial de la Salud define la violencia como el uso intencional de la fuerza o el poder físico, de hecho o como amenaza contra uno mismo, otra persona, un grupo o comunidad que cause, o tenga probabilidades de causar, lesiones, muerte, daños psicológicos, trastornos del desarrollo o privaciones.

En los varones, los aprendizajes de género incorporan el ejercicio de poder no solo contra otros sino contra sí mismos, lo que los lleva en muchas ocasiones a pasar por alto el cuidado de su salud al no hacerse revisiones médicas, pues eso cuestionaría su hombría, fuerza y resistencia ante las situaciones adveras (Salguero, 2002). Enfermar no entra en la categoría de "hombre". Si llegan a enfermar, "ya sanarán solos -afirman-, que por eso son hombres". Vale preguntarse si los aprendizajes de género en los varones de descuidar su salud física y emocional no son formas de violencia hacia sí mismos, y si las políticas de salud toman en consideración ese hecho y si repro- 
ducen y mantienen las desigualdades de género, asumiendo una concepción estereotipada sobre los hombres y su atención médica o psicológica.

Lo anterior se vincula a los procesos de aprendizaje de género del ser hombre. ¿Cómo aprenden a ser hombres?, ¿desligándose, violentando sus cuerpos, su vida? Es posible tener algunos acercamientos desde los diferentes ámbitos por los que transitan en su trayectoria de vida.

Es en los círculos familiares donde se despliegan formas de aprendizaje no solo de lo que significa ser mujer o ser hombre, sino de los roles de género que les corresponden (Oliveira, Eternod y López, 1999; Schmukler, 1996); es ahí donde incorporan y aprenden las prácticas del cuidado de sí, como señala Foucault (1987), al considerar o no el cuidado del cuerpo, la alimentación o la vida emocional para lograr el "bien-estar". ¿Es ahí donde se incorporan como parte de la socialización y aprendizaje de los hombres? En el caso de las mujeres, se ha documentado históricamente que la ética del cuidado se ha dirigido a ellas, quienes aprenden desde pequeñas a cuidar de sí mismas y de otros; en la práctica, incorporan elementos y situaciones que conducen al cuidado de sí, no solo física sino emocionalmente, especializándose en la escucha de situaciones difíciles o conflictivas que viven los otros. Como madres, amigas, tías o vecinas, ahí están para escuchar y dar consejos, para cuidar.

En el caso de los varones -sean niños, adolescentes o adultos-, las quejas están vedadas; deben soportar y resistir no solo el dolor físico sino también el emocional por las situaciones que no pueden resolver y que, según los modelos de género, "deberían saber", pues ellos están para resolverlo todo, no para lamentarse ni para decir "me duele", "me siento mal", "estoy cansado" o "no quiero ir a trabajar, pues prefiero quedarme en casa y cuidar de mi hijo", todo lo cual sería impensable.

¿Será que el cuidado de sí se haya feminizado y que por ello los sujetos de género masculino no tengan cabida en dicho cuidado, aun cuando los costos sociales, económicos y en la salud sean muy altos, vayan en aumento y sigan cobrando vidas?

Un tema de investigación que requiere acercamiento, abordaje y análisis desde tal perspectiva son los procesos de aprendizaje de género de los hombres en relación al cuidado de sí, no solo en los ámbitos familiares sino en los escolarizados y de la salud, pues es probable que se estén reproduciendo desigualdades al no considerar una ética del cuidado en los varones. Vargas, Rodríguez y Hernández (2010) señalan que una manera de construirse como persona es a través de la autonomía al generar procesos mediante los cuales se propicie la independencia familiar, pero al mismo tiempo sabiendo que se pertenece a ese ámbito y que se es apoyado por él. Habría que identificar procesos de construcción de autonomía en los varones en los que se incorpore el cuidado de sí como una forma de "ser persona", de estar en el mundo desde el "bien-estar", identificando situaciones de riesgo, como el abuso de alcohol y drogas o el trabajo excesivo, que los coloca en situaciones de riesgo para la salud al violentarse ellos mismos o a través de otros. Aprender a tomar distancia de situaciones conflictivas, atreverse a romper el silencio, cuidar de sí, evitar poner en riesgo la salud o el bienestar, es muy poco común en los varones.

\section{PATERNIDAD, APRENDIZAJES Y ESTEREOTIPOS DE GÉNERO}

Existen concepciones ideológicas sobre la paternidad que en muchos casos incluyen estereotipos sobre lo que "debería ser" un padre al asignarle valoraciones positivas o negativas según su cumplimiento y responsabilidad. La madre sigue siendo considerada como la cuidadora principal de la familia, en tanto que el padre lo es solo una parte del tiempo ya que su papel principal es de proveedor económico en los estereotipos de género tradicionales (Sunderland, 2006), lo que tiene implicaciones en la vida y en las condiciones de salud, pues los hombres están más preocupados por la proveeduría y el trabajo que por la relación familiar y el tiempo dedicado a los hijos. ¿Existe la posibilidad de ser un padre diferente, comprometido, amoroso y sensible a las necesidades de sus hijos más allá de la mera proveeduría económica? Si así fuera, ¿su pareja y familia le darían el respeto, la tolerancia y la aceptación de sus ideas, creencias y organización de vida de ese hombre, o cuestionarían su actuación con base en los estereotipos 
de género, llegando incluso a cuestionarlo como hombre? Algunos padres que se atreven a ser diferentes reciben críticas por transgredir el "deber ser", según el cual los hombres se comprometen poco con la familia, no pasan mucho tiempo con los hijos, y es su esposa quien debe hacerse cargo de las labores de la casa. Atreverse a ser un hombre o padre "diferente" genera incertidumbre, y en ocasiones conflicto, pues confronta y atenta contra lo establecido y pone en entredicho las maneras en que se supone deben ser los hombres. Enfrentar una postura distinta más allá de los estereotipos establecidos es, pues, conflictivo.

Ser padre forma parte de un proceso sociocultural complejo que se va construyendo momento a momento en la relación compartida con la pareja y los hijos (Salguero, 2007a). Construir una identidad como padre adquiere un carácter relacional y temporal en los escenarios y en las prácticas en que participan los hombres que han decidido serlo. En el caso de la paternidad, los varones se enfrentan a una búsqueda de identidad que implica un esfuerzo por encontrar puntos de referencia que les permitan comprender su actuación en un ámbito que en términos generales les resulta desconocido. Salguero y Pérez (2008) proponen analizar este proceso con el apoyo de una metáfora: los padres como inmigrantes en un terreno desconocido, ya que las prácticas tradicionales vividas con sus propios padres, caracterizadas en muchas ocasiones por una escasa participación en las decisiones reproductivas y en la crianza de los hijos, son cuestionadas hoy día. Algunos se han preguntado por esas "nuevas" formas de actuación refiriendo que con la paternidad entran a un terreno desconocido que no saben cómo afrontar, que no encuentran referentes concretos, que tienen que ir ensayando y construyendo día con día nuevas formas de relación, sin saber si lo que hacen como padres es lo correcto. Ello implica la necesidad de aprender a ser padres en ese territorio inexplorado, construir una postura, buscar información y usarla en la práctica. La metáfora del inmigrante permite resaltar que hay una gran variedad de cuestiones que tienen que negociarse para saber cómo habitar ese terreno ignoto donde nada está resuelto o definido de antemano, y es a partir de esas múltiples negociaciones que se va construyendo una identidad que se improvisa con lo que la experiencia ha incorporado y con los recursos disponibles en los escenarios socioculturales (cf. Holland, Lanchicotte, Skinner y Cain, 1998).

Las distintas formas de improvisar dan como resultado una manera de ubicarse y experimentar ese terreno novedoso y de construir una identidad como padre. Para Hoghughi y Long (2004), la paternidad integra actividades y procesos que involucran a los hijos, la madre y otros familiares, quienes tienen diferentes concepciones de sí mismos y de los demás que en ocasiones generan conflictos y tensiones entre ellos.

Analizar lo que los hombres viven como padres y su relación con la salud física o emocional es importante pero escasamente documentado. Según la información disponible, "ser padre" es un concepto con una enorme carga de valor y responsabilidad; no es suficiente con ser padre, sino que existe una constante preocupación por ser "buen" padre, esto es, tener paciencia y disponibilidad y atender las necesidades de los hijos. El problema es que no existe acuerdo acerca de qué debería hacerse para lograrlo, lo que genera incertidumbre y preocupación en los padres, llevándolos a una búsqueda constante, a ensayos múltiples y a una frecuente improvisación en el proceso, todo lo cual genera ansiedad, temores, miedos y en ocasiones depresión.

Ser padre significa para algunos construir formas de relación distintas a las que aprendieron de sus padres, confrontando sus temores y miedos y conduciéndolos a construir y reconstruir la trayectoria de vida en la que se accede y se vive la paternidad, la que forma parte del proceso de aprendizaje de ser padres en la relación con sus hijos. De cualquier manera, es posible decir que un hijo enseña mucho al padre y siempre le dice cómo actuar. La satisfacción y emoción de amar y participar en la crianza de los hijos, de compartir el tiempo y disfrutar la relación, da un sentido diferente a los varones, muchos de los cuales llegan a afirmar que ha sido lo más importante que han hecho en su vida. La paternidad genera un proceso de cambio y resignificación por sobre los estereotipos centrados en el poder, la autoridad, la ausencia, el distanciamiento y la escasa participación de los varones en lo que concierne a los hijos. Sin embargo, las instituciones y ámbitos laborales no toman en consideración esta posibilidad, sino que 
la idea sigue siendo ver a los hombres únicamente como engranajes de la industria, del trabajo, sin vida personal, lo que ha sido y es reforzado mediante los procesos de socialización genérica.

El riesgo de los procesos de socialización y aprendizajes de género es la creencia estereotipadas de que ellos solo son para el trabajo. Desde temprana edad, el mundo del trabajo influye en la vida de los hombres y forma parte de su identidad; la idea es que deben trabajar, pues es a través de esta tarea que obtendrán un lugar y serán reconocidos como hombres (Salguero, 2007b), lo que es incompatible con el cuidado, la crianza y la atención de los hijos, lo que requiere tiempo y los enfrenta a dilemas y conflictos entre lo que se quiere y las posibilidades que se tienen para hacerlo, pues con frecuencia, aunque quisieran destinar más tiempo a sus hijos, los horarios de trabajo no lo permiten. La exigencia social de género es que sean proveedores, que lleven dinero a casa, y la forma de lograrlo es trabajando (Cape1la, 2007; Salguero, 2009). No obstante, el trabajo implica en ocasiones horarios extendidos que se convierten para algunos en una permanente fuente de conflicto, pues la convivencia familiar se ve obstaculizada o definitivamente impedida.

El no poder estar con sus hijos produce a los hombres una sensación de insatisfacción, conflicto y en ocasiones depresión. Montiel, Salguero y Pérez (2008) plantean que una posibilidad de resolver el conflicto es a través de las negociaciones y acuerdos que establecen con su pareja para conciliar la relación trabajo-familia y balancear los tiempos y actividades. Pero como no siempre es posible, hay conflictos permanentes en la pareja e incluso con los hijos, lo que acrecienta los niveles de estrés, angustia y aislamiento, por lo que muchos reasumen el papel que refrenda la masculinidad hegemónica y optan por el trabajo, con lo que la presión ejercida por aquellos será mayor.

El desempleo o el trabajo informal con bajos ingresos se convierten en una desventaja que frecuentemente da al traste con la relación. En ocasiones, sus parejas condicionan la posibilidad de ver a sus hijos. Al preguntársele a un hombre cuál había sido la situación más difícil que había vivido, dijo que ello ocurrió cuando se quedó sin trabajo y la esposa le prohibió ver a sus hijos hasta que llevara dinero, lo que le generó un estado de ansiedad constante que le paralizó el brazo. "Sentía que moría -comentó- no por el dolor físico sino emocional al no poder ver a mis hijos, pues aunque buscaba trabajo no lo podía encontrar".

Condicionar la convivencia con los hijos a la proveeduría genera dolor y sufrimiento, angustia, estrés, ansiedad y depresión, lo que representa una condición de riesgo para la salud emocional y psicológica de los padres varones (Bolaños, 2014). Como ya se ha señalado antes, los aprendizajes de género incorporan estereotipos según los cuales se evalúa la actuación de los hombres como la responsabilidad y el cumplimiento del rol de proveedores económicos. Si tienen trabajo y obtienen recursos económicos suficientes, serán valorados como hombres responsables; si no, como irresponsables. "Ya no son hombres", sin importar lo que puedan pensar o sentir o las implicaciones que ello tiene en sus condiciones de salud.

La relación entre la masculinidad, la paternidad y los aprendizajes de género en los hombres lleva a considerar los estereotipos y el daño que generan en los procesos de socialización y el aprendizaje de ser hombre, de ser padre, al construir identidades conflictivas y tensionadas que imponen variados riesgos a la salud física y emocional al tratar los hombres de alcanzar los estándares bajo los que se miden y evalúan en el mundo social.

\section{CONCLUSIONES}

Los aprendizaje de género como hombres y padres incorporan estereotipos centrados en la fortaleza, la responsabilidad, el cumplimiento, creando así expectativas, actividades y formas de relación consideradas propias de ellos y de la paternidad. La responsabilidad económica y la proveeduría son en México cada vez más difíciles para los hombres en la medida en que los cambios socioculturales han favorecido el ingreso de las mujeres a los ámbitos laborales, lo que no implica que las mujeres generen condiciones de desventaja social en perjuicio de los hombres, sino que las condiciones de trabajo no se han desarrollado de manera tan equitativa para que tengan cabida a hombres y mujeres. La distribución geográfica en las ciudades implica muchas horas de traslado entre 
los hogares y los centros de trabajo, de modo que las posibilidades reales de convivencia con la familia y con los hijos son cada vez más difíciles y complicadas.

En numerosas familias el padre es avasallado por el trabajo; sin posibilidad de convivir, su rol se centra en esa actividad y en la de ser proveedor; de hecho, es su manera de existir. Sin embargo, no poder cumplir con ese rol no significa irresponsabilidad o carecer de hombría, lo cual se convierte en una forma de desigualdad social; por ende, es necesario identificar tensiones, contradicciones y malestares en los procesos de aprendizaje como hombres y como padres. Figueroa (2010) y Figueroa y Nájera (2015) consideran que se ha hecho cada vez más evidente la necesidad de generar información sobre la población masculina para considerar sus responsabilidades y derechos en los procesos reproductivos. En ese sentido, es importante dar cuenta de sus experiencias en contextos como el trabajo y la familia (Bolaños, 2014; Figueroa, Jiménez y Tena, 2006).

Si bien se han generado líneas de investigación sobre los procesos de aprendizaje de los hombres y de la paternidad, se ha cuestionado la pertinencia o no de usar ciertas categorías para denotar algunas experiencias reproductivas de los varones (Guevara, 2003), pues en el caso de la paternidad se enfrentan a un aprendizaje complejo a fin de satisfacer los requerimientos de tiempo, formas de relación, responsabilidad y cumplimiento, lo que no se atreven a expresar por el peligro que entraña caer fuera del modelo normativo de la masculinidad, convirtiéndose así en situaciones de riesgo para la salud. Shepard (1996) considera que los hombres tienen derecho a cuestionar los atributos que socialmente se han construido sobre los roles que deben cumplir a partir de los estereotipos de género.
Se hace necesario investigar los malestares derivados de los procesos de aprendizaje de género de los hombres, los cuales incorporan estereotipos que dañan el cuidado de su salud. Figueroa (2010) considera que por lo general los hombres hablan poco de sus malestares y de su incomodidad ante las consecuencias de tales estereotipos, no únicamente de ellos sino de las propias mujeres.

En fin, el proceso de construcción de la masculinidad incorpora una visión estereotipada de lo que se supone debe ser un hombre, tal como señalan Olavarría, Benavente y Mellado (1998), aunque los costos de vivir como hombres a partir de ese modelo hegemónico sean muy altos (cf. Connell, 2015). Al respecto, Tena y Jiménez (2014) plantean que los hombres pueden estar viviendo situaciones contradictorias, ambivalentes, pero que, por el lenguaje asociado al estatus social que ocupan, es factible que ni siquiera las identifiquen o que incluso explícitamente se rehúsen a nombrarlas como tales. Por ello, es importante documentar las necesidades, molestias e inconformidades de los varones a fin de elaborar estrategias que puedan ayudar a contrarrestarlas al redefinir sus responsabilidades en los espacios reproductivos y relacionales, y en ese sentido afirmar un concepto de salud que vaya más allá de lo físico al considerar la parte emocional, pues en ocasiones se relaciona con procesos depresivos y ansiedad, pérdida de autoestima y sentido de pertenencia.

Es importante abrir espacios donde los hombres puedan hablar sobre esa parte conflictiva que representa la enorme responsabilidad que históricamente se les ha asignado: cargar sobre sus hombros el peso total de la familia, lo que se ha convertido en un riesgo para la salud.

\section{REFERENCIAS}

Bolaños, F. (2014). El Grupo de "Apoyo Emocional al Desempleo" en hombres: Resultado de investigación. En J. G. Figueroa (Coord.): Políticas públicas y la experiencia de ser hombre. Paternidad, espacios laborales, salud y educación (pp. 111-174). México: El Colegio de México.

Camussi, E. y Leccardi, C. (2005). Stereotypes of working women: the power of expectations. Social Science Information, 44(1), 113-140.

Capella R., S. (2007). ¿Sólo trabajadores/proveedores? En G. Jiménez L. y O. Tena (Coords.): Reflexiones sobre masculinidades y empleo (pp. 153-180). México: UNAM. 
Connell, R. (2015). Masculinidades. México: UNAM.

Figueroa J., G. (2001). La soledad en la paternidad. Fem, 25(218), 15-19/48.

Figueroa J., G. (2010). La construcción de la titularidad para el ejercicio de los derechos reproductivos. En B. García y M. Ordorica (Coords.): Población (t. I: Los grandes problemas de México) (pp. 253-289). México: El Colegio de México.

Figueroa J., G., Jiménez, L. y Tena, O. (2006). Ser padres, esposos e hijos: prácticas y valoraciones de varones mexicanos. México: El Colegio de México.

Figueroa J., G. y Nájera J., N. (2015). El uso de las autopsias verbales para analizar algunos suicidios de varones progenitores. Acta Universitaria, 25(NE-3), 19-25. Doi: 10.15174/au.2015.848.

Foucault, M. (1987). Historia de la sexualidad (1: La voluntad de saber) (15 ed.). México: Siglo XXI Editores.

Fuller, N. (2000). Significados y prácticas de paternidad entre varones urbanos del Perú. En N. Fuller (Ed.): Paternidades en América Latina (pp. 35-90). Lima: Pontificia Universidad Católica del Perú/Fondo Editorial.

Guevara, E. (2003). ¿Se puede hablar de los derechos reproductivos de los hombres en el caso del aborto? Memorias del VI Coloquio del Programa Interdisciplinario de Estudios sobre la Mujer. México: El Colegio de México (mimeo), México, D.F., 13 al 15 de marzo.

Hoghughi, M. y Long, N. (2004). Key concepts. Parenting: an introduction. Handbook of Parenting (pp. 1-18): New Delhi: SAGE.

Holland, D., Lanchicotte, W., Skinner, D. y Cain, C. (1998). Identity and agency in cultural worlds. Cambridge, MA: Harvard University Press.

Montiel, P., Salguero, A. y Pérez, G. (2008). El trabajo: ¿fuente de conflicto en el ejercicio de la paternidad? Psicología y Ciencia Social, 10(1), 26-40.

Nolasco, S. (1989). O mito da masculinidad. Río de Janeiro: Roco.

Olavarría, J., Benavente, C. y Mellado, P. (1998). Masculinidades populares. Varones adultos jóvenes de Santiago. Santiago de Chile: FLACSO.

Oliveira, O., Eternod, M. y López, P. (1999). Familia y género en el análisis demográfico. En B. García (Coord.): Mujer, género y población en México. México: El Colegio de México/Sociedad Mexicana de Demografía.

Organización Panamericana de la Salud (2002). La violencia, un problema ubicuo. En OPS (Ed.): Informe mundial sobre la violencia y salud. Ginebra: Organización Mundial de la Salud.

Parsons, T. (1881). Definitions of health and illness in the light of American values and social structure. Concepts of health and disease. En G. E. Jaco (Ed.): Patients, physicians and illness. New York: Free Press (reprinted in Social Structure and Personality. London: Routledge and Kegan Paul).

Rivera, R. y Ceciliano, Y. (2005). Cultura, masculinidad y paternidad: las representaciones de los hombres en Costa Rica (2 ${ }^{\mathrm{a}}$ ed.). San José de Costa Rica: FLACSO.

Rodríguez M., E. (2001). Experiencias del trabajo de masculinidades en Bolivia. En J. G. Figueroa y R. Nava (Eds.): Sexualidad, salud y reproducción. Memorias del Seminario-Taller Identidad Masculina, Sexualidad y Salud Reproductiva (Documento de trabajo No. 4, pp. 32-36). México: El Colegio de México.

Salguero, A. (2002). Significado y vivencia de la paternidad en el proyecto de vida de los varones. Tesis de Doctorado en Sociología. México: UNAM.

Salguero, A. (2007a). Más allá de la obviedad..., la paternidad, un proceso histórico, sociocultural y de aprendizaje. En A. Salguero, D. Córdoba y S. Sapién (Coords.): Reproducción y paternidad (pp. 5-44). México: UNAM.

Salguero, A. (2007b). El significado del trabajo en las identidades masculinas. En L. Jiménez, y O. Tena (Coords.): Reflexiones sobre masculinidades y empleo (pp. 429-448). México: UNAM/Centro Regional de Investigaciones Multidisciplinarias.

Salguero, A. (2009). Ser proveedor no es suficiente: reconstrucción de la identidad en los varones. Revista Internacional de Estudios sobre Masculinidades, 4(7), octubre-diciembre.

Salguero, A. y Pérez, G. (2008). La paternidad en los varones: una búsqueda de identidad en un terreno desconocido. Algunos dilemas, conflictos y tensiones. Revista Internacional de Estudios sobre Masculinidades, 3(4).

Sánchez G., M. (2000). El concepto de salud: análisis de sus contextos, sus presupuestos y sus ideales. Cuadernos del Programa Regional de Bioética, 7. Santiago de Chile: Programa Regional de Bioética para América Latina y el Caribe OPS/OMS.

Schmukler, B. (1996). La socialización de los niños y las relaciones de género en la familia. En J. G. Figueroa P. (Coord.): Elementos para un análisis ético de la reproducción (pp. 243-258). México: UNAM.

Shepard, B. (1996). La masculinidad y el rol masculino en la salud sexual. En A.A.: Salud reproductiva: nuevos desafios (pp. 73-86). Lima: Universidad Peruana Cayetano Heredia.

Sunderland, J. (2006). 'Parenting' or 'mothering'? The case of modern childcare magazines. Discourse \& Society, 17(4), 503-527.

Tena, O. y Jiménez, L. (2014). Algunos malestares en la experiencia de los varones: ¿podemos ir reflexionando sobre sus derechos sexuales y reproductivos? En J. G. Figueroa y A. Salguero (Coords.): ¿Y si hablas de tu ser hombre? Violencia, paternidad, homoerotismo y envejecimiento en la experiencia de algunos varones (pp. 331-358). México: El Colegio de México.

Vargas, J., Rodríguez, M. y Hernández, M. (2010). La diferenciación del yo y la relación hacia la violencia en el varón. Revista Electrónica de Psicología Iztacala, 13(4), 197-216. 